\title{
A case of Lyme disease in a Japanese woman
}

This article was published in the following Dove Press journal:

Infection and Drug Resistance

\author{
Masafumi Seki' \\ Yuji Watanabe ${ }^{2}$ \\ Hiroki Kawabata ${ }^{3}$ \\ 'Division of Infectious Diseases and \\ Infection Control, Tohoku Medical and \\ Pharmaceutical University Hospital, \\ Sendai City, Miyagi, Japan; ${ }^{2}$ Laboratory \\ for Clinical Microbiology, Tohoku \\ Medical and Pharmaceutical University \\ Hospital, Sendai City, Miyagi, Japan; \\ ${ }^{3}$ Department of Bacteriology-I, \\ National Institute of Infectious \\ Diseases, Tokyo, Japan
}

\begin{abstract}
This report presents the case of a Japanese patient who developed Lyme disease. The patient was a 44-year-old woman who had general fatigue and the inability to open her mouth. She was initially suspected of having mild tetanus with lockjaw; however, she reported a past history of a tick bite while camping in the USA and had erythema migrans 2 months before this visit. Finally, Lyme disease was serologically confirmed. A few cases of Lyme disease are annually reported in Japan; however, this infectious disease should also be suspected.

Keywords: Borrelia burgdorferi, Clostridium tetani, tetracycline, facial nerve palsy, erythema migrans
\end{abstract}

\section{Introduction}

Lyme disease is caused by the bacterium Borrelia burgdorferi and is transmitted to humans through the bite of infected blacklegged ticks. ${ }^{1}$ Typical symptoms include fever, headache, fatigue, and a characteristic skin rash called erythema migrans. Untreated Lyme disease can produce a wide range of symptoms according to the stage of infection, such as fever, rash, arthritis, and facial paralysis. Moreover, some patients progress to the chronic type of Lyme disease. ${ }^{2}$

In Japan, Lyme disease is rare in areas outside of the northern islands (e.g., Hokkaido area). Therefore, Lyme disease may not be included in the differential diagnosis of fever of unknown origin and/or facial paralysis in general medicine outpatient clinics in Japan. ${ }^{3,4}$

Here, we present a case of Lyme disease in a patient with facial paralysis and fatigue, which was initially suspected as mild tetanus with lockjaw in the general ward.

\section{Case report}

A 44-year-old woman presented at the neurological outpatient clinic in July 2017 with mild mouth paralysis and mild fatigue that had persisted for 2 months. She had no medical history, but primarily resides in New York in the USA since her marriage about 10 years ago. She recently returned to Japan to see her parents, 3 days before visiting the clinic. Physical examination indicated the following: temperature of $37.2^{\circ} \mathrm{C}$, blood pressure of $120 / 60 \mathrm{mmHg}$, respiratory rate of 18 breaths/min, and consciousness level of E4V5M6 on the Glasgow Coma Scale. There were no crackles (rhonchi) in either lung field; however, her oral muscle was slightly rigid. The neurologist initially suspected mild tetanus with lockjaw caused by Clostridium tetani infection, which is relatively common in our area, and consulted with our infectious diseases (ID) division. She was subsequently admitted to our department. Her initial white blood cell
Correspondence: Masafumi Seki Division of Infectious Diseases and Infection Control, Tohoku Medical and Pharmaceutical University Hospital, I-I2-I Fukumuro, Miyagino-ku, Sendai City, Miyagi 983-8612, Japan

Tel +8I 22983 I22 I

Fax +8I 229830507

Email seki@hosp.tohoku-mpu.ac.jp 
count was 6400/L (Neu 79\%, Lymph 10\%, Mono 8\%, and Eo 3\%), and her C-reactive protein level was $0.58 \mathrm{mg} / \mathrm{dL}$. She could open her mouth about $1.5 \mathrm{~cm}$, but her left side of the mouth sagged unilaterally, and she could hardly close her left eye. Therefore, gross examination of her mouth suggested facial paralysis rather than lockjaw (image unavailable due to the patient's refusal). We then asked again about her living conditions, and she revealed a past history of tick bite while camping in Pennsylvania 3 months previously. She also noted temporary characteristic skin changes with slight itching and pain on her right limb about 1 week after camping, which might be so-called erythema migrans (Figure 1). This skin lesion persisted for 1-2 weeks.

According to the laboratory findings and past history, we suspected Lyme disease and administered oral minocycline therapy (100 mg, twice/day), which was more available rather than doxycycline in Japan, for about 2 weeks. Steroids were not used. The patient's symptoms, including fatigue, mouth sagging, and hardly closed eye, subsequently improved, and serum testing was positive for anti-Borrelia antibodies (Figure 2).

\section{Discussion}

Lyme disease is a multisystemic disorder caused by infection with spirochete species including B. burgdorferi. ${ }^{1}$ The vectors of human borreliosis are ticks (Ixodes species), and Ixodes persulcatus is a transmission vector in Japan. ${ }^{3}$ On the other hand, the reported number of Lyme disease cases is less than 20 per year. Therefore, Lyme disease is not generally considered a common cause of fever of unknown origin (FUO) in Japan, although it is very common in North America and Europe. However, some sporadic and transported cases have been reported in mainland Japan. Thus, Japanese ID physicians should consider this disease as one of the possible causes of FUO and carefully ask patients about their living conditions and past histories. ${ }^{4,5}$

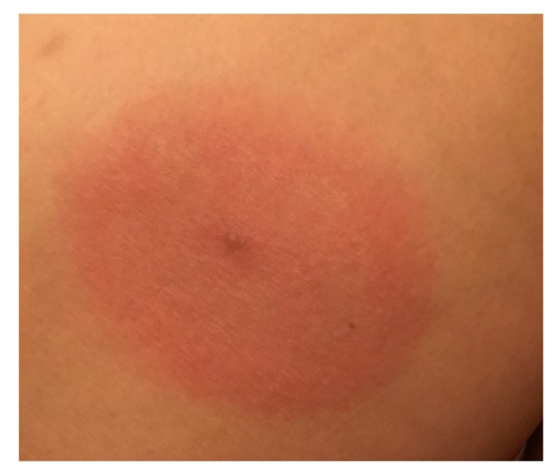

Figure I Skin erythema migrans seen in the present patient (The photo was kindly provided by the patient).
In the present case, the patient had a persistent facial paralysis and fatigue after camping in the USA and visited our hospital in Japan. Tetanus was initially suspected, which is a

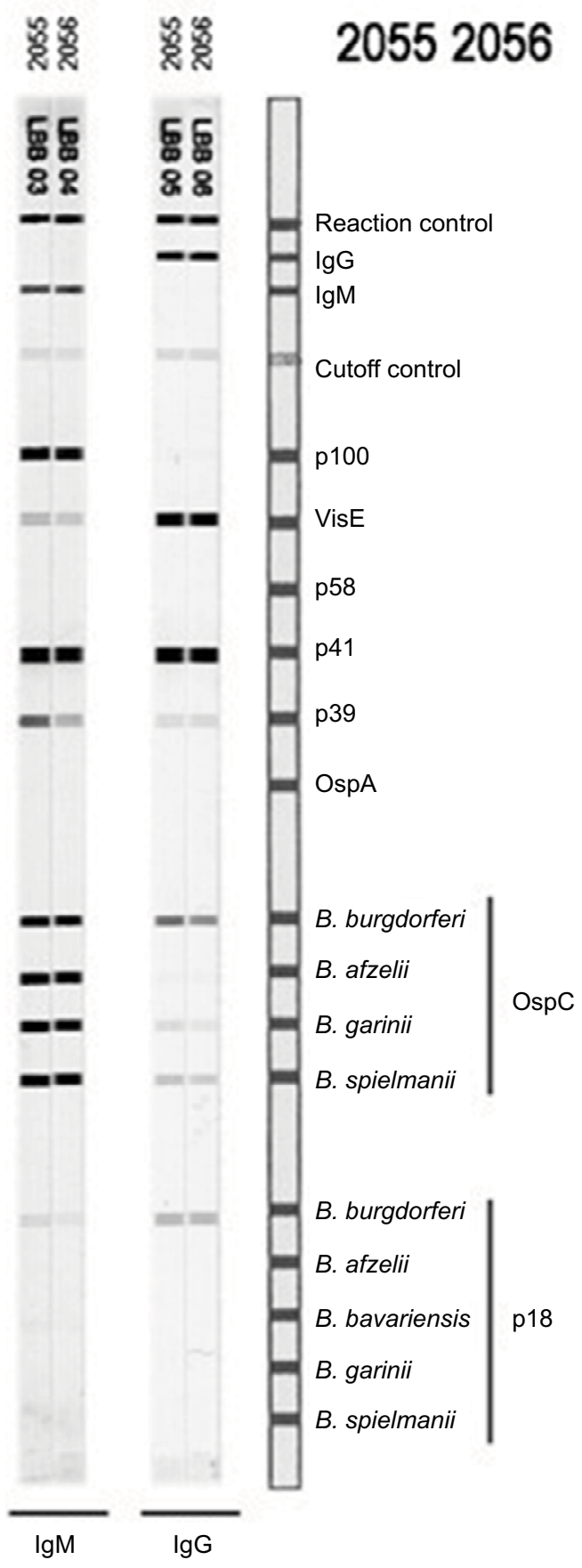

Figure 2 Anti-Borrelia antibodies detected by recomLine Borrelia IgG and IgM strip immunoassays (Mikrogen Diagnostik, Neuried, Germany). Sera 2055 and 2056 were collected in July and August 2017, respectively. The patient's sera reacted to various recombinant antigens as follows; pl00 (93 kDa antigen), VlsE, p4I (4I kDa antigen, Flagellin), p39 (BmpA), OspCs (2l-24 kDa antigen), and pl8 (I8 kDa antigen). The test result suggested the patient was serologically IgM and IgG positive for Lyme disease (https://www.mikrogen.de/uploads/tx_oemikrogentables/dokumente/ GARLBB007EN.pdf). The patient also tested IgM positive for Lyme disease by the Center for Disease Control and Prevention recommendations.

Note: Recommended reading: Test Performance and Interpretation from the Second National Conference on Serologic Diagnosis of Lyme Disease. MMWR. 1995;44(31):590-591.

Abbreviations: IgG, immunoglobulin G; IgM, immunoglobulin M. 
relatively common disease in our area, because the inability to open her mouth was considered to be lockjaw rather than facial paralysis caused by Lyme disease. Given that Lyme disease and very mild tetanus have overlapping symptoms, Lyme disease may not be initially considered in Japan.

Lyme disease is defined as the attribution of various atypical syndromes to protracted B. burgdorferi infection, sometimes showing chronic pain, fatigue, and neurocognitive and behavioral symptoms, as well as various alternative medical diagnoses, which are most commonly neurologic and rheumatologic diseases, including multiple sclerosis, demyelinating diseases, amyotrophic lateral sclerosis, neuropathies, dementia, fibromyalgia, and chronic fatigue syndrome. ${ }^{2,6}$ These diseases may be differential diagnosis of the Lyme diseases and this patient, but not considered because they are out of infection and acute onset on the previously healthy young adults.

Therefore, a past history of tick bite in a forested area and other objective findings, such as erythema migrans, become more important than subjective findings in the diagnosis of Lyme disease. In Japan, Lyme disease in humans is caused by infection with $B$. garinii or $B$. afzelii, which are biologically and specifically transmitted by I. persulcatus. ${ }^{7}$ However, this tick species is generally restricted to Hokkaido, and Japanese spotted fever is more frequently considered as a tick-related disease than Lyme disease. ${ }^{8}$ Although tick-related infectious diseases result in characteristic skin lesions, erythema migrans is unique to Lyme disease. ${ }^{9}$ In fact, in the present patient, the past history of a tick bite and appearance of possible erythema migrans strongly suggested Lyme disease. We unfortunately could not perform a biopsy of the skin rash and erythema for histopathology and polymerase chain reaction diagnosis; however, these diagnostic process may be considered as one of the novelty of their case study.

Although this patient did not receive antibiotic treatment after being bitten by a tick while camping in the USA, she had a relatively mild, chronic status, suggested the chronic type of Lyme diseases, which is a poorly defined term that describes the attribution of various atypical syndromes to protracted B. burgdorferi infection. ${ }^{2}$ In North America and Europe, treatment typically includes oral doxycycline and other antibiotics, such as penicillin and ceftriaxone. ${ }^{1}$ In the present case, 14 days of oral minocycline treatment showed an effect, and fatigue, mouth sagging, and slight disability of the eye opening disappeared. ${ }^{10}$

In summary, we diagnosed Lyme disease in a Japanese patient who generally resides in the USA and had a history of a tick bite while camping 3 months before visiting our department. Serological testing confirmed infection by Borrelia species, most possibly B. burgdorferi, and the characteristic finding of erythema migrans was also observed. The patient's mild but persistent symptoms improved after a 2-week treatment with oral minocycline. Although Lyme disease is very rare in mainland Japan, ID physicians should consider Lyme disease in the differential diagnosis of FUO with facial palsy.

\section{Acknowledgments}

This work was supported by a Grant-in-Aid for Scientific Research (17K09623 to M.S.) from the Japanese Society for the Promotion of Science. Written informed consent has been provided by the patient to have the case details and any accompanying images published.

\section{Disclosure}

The authors report no conflicts of interest in this work.

\section{References}

1. Steere AC. Lyme disease. N Engl J Med. 1989;321:586-596.

2. Lantos PM. Chronic Lyme disease. Infect Dis Clin North Am. 2015;29:325-340.

3. Murase Y, Konnai S, Githaka N, et al. Prevalence of Lyme borrelia in Ixodes persulcatus ticks from an area with a confirmed case of Lyme disease. J Vet Med Sci. 2013;75:215-218.

4. Kutsuna S, Kawabata H, Ohmagari N. Imported Lyme disease. Intern Med. 2015;54:691.

5. Iwata K, Shimada T, Kawabata H. A case of lyme disease requiring over 1 year to diagnose at an infectious-disease clinic. Kansenshogaku Zasshi. 2013;87:44-48.

6. Steere AC, Taylor E, McHugh GL, Logigian EL. The overdiagnosis of Lyme disease. JAMA. 1993;269:1812-1816.

7. Nakao M, Miyamoto K. Susceptibility of Ixodes persulcatus and I. ovatus (Acari: Ixodidae) to Lyme disease spirochetes isolated from humans in Japan. J Med Entomol. 1994;31:467-473.

8. Seki M, Ikari N, Yamamoto S, et al. Severe Japanese spotted fever successfully treated with fluoroquinolone. Intern Med. 2006;45:1323-1326.

9. Nadelman RB, Wormser GP. Reinfection in patients with Lyme disease. Clin Infect Dis. 2007;45:1032-1038.

10. Wormser GP, Ramanathan R, Nowakowski J, et al. Duration of antibiotic therapy for early Lyme disease. A randomized, double-blind, placebocontrolled trial. Ann Intern Med. 2003;138:697-704. 


\section{Publish your work in this journal}

Infection and Drug Resistance is an international, peer-reviewed openaccess journal that focuses on the optimal treatment of infection (bacterial, fungal and viral) and the development and institution of preventive strategies to minimize the development and spread of resistance. The journal is specifically concerned with the epidemiology of antibiotic resistance and the mechanisms of resistance development and diffusion in both hospitals and the community. The manuscript management system is completely online and includes a very quick and fair peerreview system, which is all easy to use. Visit http://www.dovepress.com/ testimonials.php to read real quotes from published authors.

Submit your manuscript here: https://www.dovepress.com/infection-and-drug-resistance-journal 